Revue internationale P.M.E.

Économie et gestion de la petite et moyenne entreprise

\title{
Les PME et les risques de la sous-traitance industrielle à l'heure de l'assurance qualité
}

\author{
Jean-Guy Belley, André Billette, Louis Rasmussen et Robert M. Thompstone
}

Volume 8, numéro 2, 1995

URI : https://id.erudit.org/iderudit/1008349ar

DOI : https://doi.org/10.7202/1008349ar

Aller au sommaire du numéro

Éditeur(s)

Presses de l’Université du Québec

ISSN

0776-5436 (imprimé)

1918-9699 (numérique)

Découvrir la revue

Citer cet article

Belley, J.-G., Billette, A., Rasmussen, L. \& Thompstone, R. M. (1995). Les PME et les risques de la sous-traitance industrielle à l'heure de l'assurance qualité. Revue internationale P.M.E., 8(2), 49-77. https://doi.org/10.7202/1008349ar
Résumé de l'article

Une étude empirique des relations de l'entreprise multinationale Alcan avec cinq PME du Saguenay-Lac-Saint-Jean (Québec) montre que l'implantation de l'assurance qualité change considérablement les objectifs de la sous-traitance industrielle. Les acheteurs du donneur d'ordre et les entrepreneurs soustraitants doivent concevoir différemment les risques de la sous-traitance et les moyens de s'en prémunir. Malgré les incohérences de la phase d'implantation, les PME doivent acquérir une compréhension réaliste de la stratégie poursuivie par le donneur d'ordre et des possibilités pour elles d'en tirer profit. Elles doivent aussi se préparer à négocier les ajustements structurels requis par la nouvelle coordination interfirmes et prendre conscience des risques que comporte l'assurance qualité en tant que système-expert.
Ce document est protégé par la loi sur le droit d'auteur. L'utilisation des services d’Érudit (y compris la reproduction) est assujettie à sa politique d'utilisation que vous pouvez consulter en ligne.

https://apropos.erudit.org/fr/usagers/politique-dutilisation/ 


\section{Les PME et les risques de la sous-traitance industrielle à l'heure de l'assurance qualité'}

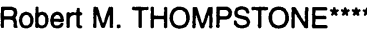

Société d'électrolyse et de chimie Alcan ltée

MOTS CLÉS

\section{Sous-traitance - Assurance qualité \\ Coordination interfirmes - Risques stratégiques}

\section{RÉSUMÉ}

Une étude empirique des relations de l'entreprise multinationale Alcan avec cinq PME du Saguenay-Lac-Saint-Jean (Québec) montre que l'implantation de l'assurance qualité change considérablement les objectifs de la sous-traitance

1. L'étude empirique à la base de cet article a été réalisée grâce à une subvention du CRSH. Les données ont été recueillies sous la promesse de la plus stricte confidentialité. La synthèse des informations et l'interprétation de leur portée sociologique relèvent entièrement de la responsabilité personnelle des auteurs. Elles ne doivent aucunement être considérées comme approuvées par l'entreprise Alcan ou par les firmes sous-traitantes qui ont accepté de participer à la recherche. Nous remercions les évaluateurs anonymes de la revue pour leurs précieux commentaires.

* Jean-Guy Belley est docteur en sociologie juridique, professeur titulaire à la Faculté de droit de l'Université Laval, Québec. Adresse: Faculté de droit, Université Laval, Sainte-Foy, Québec, G1K 7P4.

** André Billette est docteur en sociologie, professeur titulaire, Département de sociologie, Université Laval, Québec. Adresse : Département de sociologie, Faculté des sciences sociales, Université Laval, Sainte-Foy, Québec, G1K 7P4.

*** Louis Rasmussen est étudiant au doctorat en sociologie économique au Département de sociologie de l'Université Laval, Québec. Adresse : 135, rue Derfla, app. 2, La Baie, G7B 3J3.

**** Robert M. Thompstone (Ph.D. en génie de systèmes) est coordonnateur principal de l'assurance qualité, Société d'électrolyse et de chimie Alcan ltée, Jonquière, Québec. Il est également chargé de cours en gestion et assurance de la qualité à l'Université du Québec à Chicoutimi. Adresse: Société d'électrolyse et de chimie Alcan ltée, Services de l'approvisionnement, Sécal, C.P. 1500, Jonquière, G7S 4L2. 
industrielle. Les acheteurs du donneur d'ordre et les entrepreneurs soustraitants doivent concevoir différemment les risques de la sous-traitance et les moyens de s'en prémunir. Malgré les incohérences de la phase d'implantation, les PME doivent acquérir une compréhension réaliste de la stratégie poursuivie par le donneur d'ordre et des possibilités pour elles d'en tirer profit. Elles doivent aussi se préparer à négocier les ajustements structurels requis par la nouvelle coordination interfirmes et prendre conscience des risques que comporte l'assurance qualité en tant que système-expert.

\section{ABSTRACT}

An empirical study of the relations between the multinational Alcan and five SBF of the Saguenay-Lac-Saint-Jean area (Quebec) shows that the objectives of industrial subcontracting are significantly modified by the implementation of quality assurance. The purchasers of the client firm and the subcontractors must develop a new vision of the risks undertaken through subcontracting and of the means of protecting themselves against these risks. Notwithstanding the inconsistencies encountered at the implementation stage, the SBF will need an adequate understanding of the strategy pursued by the client and a realist appraisal of the opportunities opened to them by this strategy. The SBF will have to negotiate the structural adjustments required by the new dynamics of inter-firm coordination and must realize that quality assurance, like any other expert system, conveys its own risks.

\section{RESUMEN}

Un estudio empírico de las relaciones de la compañía multinacional Alcan con cinco PyMEs de la región de Saguenay-Lac-Saint-Jean (Quebec) muestra como la implantación del seguro de calidad modifica, de manera significativa, los objetivos de la subcontratación industrial. Los compradores de la firma contratante y los empresarios que toman el subcontrato deben pensar de manera diferente los riesgos inherentes al sistema de subcontrato y la manera de protegerse contra esos riesgos. A pesar de las incongruencias propias del período de implantación, las PyMEs deben adquirir una comprensión adecuada de la estrategia del contratante y de las ventajas que les ofrece esta estrategia. Las PyMEs deben también estar preparadas para negociar los ajustes estructurales que requiere la nueva dinámica de la coordinación entre firmas y tomar conciencia de los riesgos que comporta el seguro de calidad como sistema experto.

\section{Introduction}

Avant de s'imposer comme un slogan à la mode chez les dirigeants d'entreprises, la promotion de la qualité a fait l'objet d'une problématique de plus en plus articulée chez les spécialistes de la gestion des organisations. Cela a été particulièrement manifeste dans le domaine de la gestion des achats où la mise au point de programmes d'assurance qualité est considérée comme une composante majeure d'une stratégie générale d'approvisionnement scientifique (Leenders et al., 1993 ; Clouët, 1989). 
L'amélioration continue de la qualité des biens et services fournis à l'entreprise se trouve à la base d'une nouvelle philosophie qui incite les acheteurs à contribuer activement au développement de fournisseurs répondant spécifiquement aux besoins de l'entreprise plutôt que de se contenter de ce qu'offre le marché (Nollet et Leenders, 1991 ; Leenders et Blenkhorn, 1988). La conviction que le travail des fournisseurs, au même titre que celui des employés, peut et doit faire l'objet d'une organisation scientifique assurant une productivité croissante a suscité un regain d'intérêt pour les mécanismes de coopération interfirmes (Schonberger, 1982; Ravix, 1990). La préoccupation du contrôle des coûts de production, jointe à celle du contrôle de la qualité, incite à explorer les possibilités d' "alliance stratégique», de "partenariat», de «maillage » ou de «foisonnement» susceptibles d'augmenter le rendement des relations externes de l'entreprise. La gestion des achats se conçoit alors dans l'optique de l' «entreprise étendue » à laquelle participent les fournisseurs (Sérieyx, 1989; Archier et Sérieyx, 1984).

L'assurance qualité est une des institutions nouvelles qui incitent les acteurs économiques à investir dans un processus de transformation majeure $\mathrm{du}$ fonctionnement interne et des rapports externes de leur entreprise. Pour les PME traditionnellement engagées dans la sous-traitance industrielle, l'incitation devient un impératif de survie depuis que les grands donneurs d'ordre se dotent de politiques d'assurance qualité qui changent les critères de sélection et d'évaluation du rendement des sous-traitants (Martin, 1992; Esposito et Raffa, 1992).

L'implantation de l'assurance qualité suppose que les acheteurs et chargés de projet de l'entreprise cliente, d'une part, ainsi que la direction et les employés des PME sous-traitantes, d'autre part, adhèrent à des façons de faire plus tournées vers l'avenir et comportant des risques nouveaux. Parce qu'elle réclame une augmentation des risques calculés, l'assurance qualité, comme toutes les institutions de la modernité avancée, accroît l'anxiété des acteurs et leur besoin corrélatif de confiance dans les vertus des nouveaux modes d'action (Giddens, 1990). Faute de cette confiance, les acteurs investiront le moins possible dans les nouvelles façons de faire les choses et chercheront constamment à se ménager une possibilité de retraiter vers les anciennes habitudes.

On peut certainement concevoir que les acheteurs et les sous-traitants soient contraints de changer leurs manières de faire par des actes d'autorité à l'interne et par le recours au pouvoir de marché du client à l'externe. Ces moyens de pression sont peut-être indispensables pour vaincre les résistances initiales au changement. L'implantation de l'assurance qualité sera toutefois une dépense inutile si les principaux participants du système d'approvisionnement n'adhèrent pas tôt ou tard aux finalités de l'assurance qualité et 
n'adoptent pas durablement ses méthodes. Les difficultés de cette adhésion n'apparaîtront clairement qu' au terme d'une analyse des risques assumés par ces acteurs et des moyens qu'ils ont traditionnellement mis en œuvre pour se protéger contre ces risques.

Dans une première analyse (Belley et al., 1995), nous avons montré que la transformation recherchée ne pouvait réussir que si les acteurs impliqués apprennent à faire confiance aux procédés abstraits et décontextualisés de l'assurance qualité. Une confiance-système, suscitée et entretenue par les experts de l'assurance qualité, est indispensable. La confiance interpersonnelle, basée sur la familiarité et traditionnellement associée aux échanges économiques de longue durée ou s'effectuant dans une proximité physique, ne suffit pas à l'établissement des nouveaux rapports interfirmes. Elle peut même s'avérer incompatible avec le développement de la confiance-système requise.

Nous poursuivrons ici notre analyse en postulant que les experts de l'assurance qualité, en particulier ceux qui œuvrent au sein de l'organisation du donneur d'ordre et des PME sous-traitantes, ne peuvent gagner efficacement la confiance des autres acteurs qu'à la condition de bien comprendre la logique d'évaluation des risques qui préside aux comportements respectifs des acheteurs et des sous-traitants. Notre analyse prend appui sur l'étude empirique d'une expérience d'implantation de l'assurance qualité qu'il convient de décrire sommairement.

\section{L'étude empirique de référence}

Depuis 1988, la Société d'électrolyse et de chimie Alcan limitée (Alcan) a mis en œuvre un programme d'implantation de l'assurance qualité auprès de ses fabricants selon plans et devis, plus particulièrement dans la région du Saguenay-Lac-Saint-Jean au Québec. En novembre 1989, les responsables du Service régional de l'approvisionnement avaient expressément informé

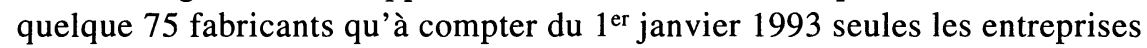
dotées d'un programme d'assurance qualité certifié par le «QMI» (Quality Management Institute) seraient invitées à soumissionner sur les appels d'offres d'Alcan. Pour contribuer à leur processus de qualification, le donneur d'ordre offrait aux sous-traitants intéressés, notamment à 33 PME du Saguenay-LacSaint-Jean, l'assistance technique du personnel relevant de son coordonnateur principal à l'assurance qualité et à l'administration de contrat.

Notre étude a été réalisée auprès de cinq PME du Saguenay-Lac-SaintJean ayant pour caractéristiques communes d'avoir obtenu leur certification et de compter parmi les principaux sous-traitants d'Alcan du point de vue de la valeur des contrats exécutés au cours des dernières années. Entre juin et 
décembre 1993, plus de soixante entretiens semi-structurés d'une à deux heures chacun ont été réalisés, d'une part, auprès des dirigeants et du personnel des cinq entreprises sous-traitantes et, d'autre part, auprès d'acheteurs, chargés de projets et administrateurs de contrat des établissements d'Alcan dans la région. Centrés sur les caractéristiques et l'évolution de la relation d'Alcan avec chacune des firmes sous-traitantes, avant et après la mise en œuvre du programme d'assurance qualité, les entretiens avaient pour but explicite de fournir des informations objectives (l'entreprise sous-traitante, son marché, la nature des contrats exécutés pour Alcan) et subjectives (qualité de la communication, évaluation des risques et bénéfices, perception de l'autre) qui permettraient de mieux connaître les conditions, les difficultés et les limites d'une évolution de la coopération interfirmes dans le sens de l'amélioration continue de la qualité et du partenariat.

Les particularités de notre étude ne nous permettent évidemment pas de prétendre avoir cerné toutes les dimensions des stratégies d'implantation de l'assurance qualité ou d'établissement du partenariat dans les rapports interfirmes. Les relations d'affaires analysées se limitent au domaine tout à fait distinct de la sous-traitance industrielle. Elles se ressentent forcément des particularités du marché des biens et services utiles à la production de l'aluminium primaire, des caractéristiques organisationnelles de l'entreprise Alcan et de la configuration socio-économique du Saguenay-Lac-Saint-Jean. Au surplus, notre étude se voulait expressément qualitative plutôt que quantitative. La possibilité d'une compréhension en profondeur du sens des choses nous paraissait préférable à la recherche d'une représentativité statistique.

Cela dit, plusieurs facteurs nous permettent de croire que notre réflexion présente un intérêt théorique et pratique, au-delà du milieu étudié, pour tous ceux qui s'intéressent à la dynamique de la sous-traitance industrielle dans le contexte nouveau créé par l'assurance qualité. Le premier de ces facteurs est l'excellente collaboration des représentants de toutes les entreprises impliquées et la richesse des informations qu'elle nous a permis de recueillir. Le deuxième facteur tient au fait que la stratégie d'implantation de l'assurance qualité mise en œuvre par Alcan a été poursuivie de manière suffisamment claire et soutenue, malgré la conjoncture économique défavorable des trois dernières années, pour que tous les acteurs concernés aient pris conscience du changement souhaité et commencé à en évaluer les enjeux et les conditions de réussite, quel que soit leur degré d'adhésion aux objectifs du programme. Enfin, la diversité des connaissances et des intérêts disciplinaires mis en commun par les membres de l'équipe de recherche a permis d'entreprendre le travail de terrain avec une solide connaissance préalable du milieu étudié, puis d'en interpréter les résultats en bénéficiant de l'éclairage complémentaire des sciences de la gestion (Thompstone, 1991), de la sociologie économique 
(Billette et Carrier, 1993 ; Billette et al., 1991 ; Rasmussen, 1992) et de la sociologie du droit des contrats (Belley, 1991, 1992, 1993).

L'expérience particulière menée par Alcan au Saguenay-Lac-Saint-Jean a servi de référence empirique à une réflexion que nous voulions aussi large que possible. Comme en témoigne le titre de l'article, on ne doit pas s'attendre à trouver ici une description détaillée de cette expérience ni un compte rendu des opinions qui ont été exprimées sous le sceau de la confidentialité. Chaque fois que cela était possible, nous avons complété ou même extrapolé nos données empiriques en ayant recours à la littérature scientifique sur l'assurance qualité et la coordination interfirmes. En revanche, les entretiens avec les acteurs de la sous-traitance nous ont permis d'appréhender dans leur réalité complexe et dynamique les problèmes que la littérature scientifique aborde souvent de façon séparée et dans une optique formelle ou même normative. L'objectif visé étant d'enrichir la compréhension des choses, il ne nous a pas semblé nécessaire de distinguer systématiquement les apports respectifs des chercheurs et des acteurs.

Une synthèse préliminaire de nos réflexions a été communiquée à une quinzaine de répondants rencontrés à l'étape de la collecte des informations. Les liens théoriques et empiriques que nous établissons ont été jugés pertinents et éclairants par les personnes consultées. Ce dialogue avec les acteurs confère à notre interprétation une vraisemblance qui ajoute, selon nous, à son intérêt scientifique (Dubet, 1994). Les entretiens réalisés et la littérature consultée nous auront donc permis d'entrevoir l'impact de l'assurance qualité sur la pratique quotidienne de la sous-traitance industrielle. Nous en décrirons d'abord les effets sur l'exercice du métier d'acheteur avant de montrer comment l'approche traditionnelle de l'entrepreneur sous-traitant est, elle aussi, appelée à se transformer.

\section{Les risques de l'acheteur}

Du point de vue organisationnel et juridique, l'acheteur est l'intermédiaire par excellence de la sous-traitance industrielle. Dans la division interne du travail, c'est à lui qu'est dévolue la responsabilité spécifique de l'aspect commercial des contrats, en particulier le choix du sous-traitant et le prix de la transaction. À titre de mandataire, il est en principe le seul à pouvoir lier contractuellement le donneur d'ordre.

Dans l'exercice quotidien de son rôle, l'acheteur assume trois risques principaux. Le premier a trait à la qualité : le produit ou le service fourni ne répond pas au besoin objectif ou ne donne pas satisfaction à l'usager ; le choix de l'acheteur lui attire des récriminations de la part des usagers, ses clients 
internes. Le deuxième risque concerne le prix payé : le contrôleur du budget ou le vérificateur externe reproche à l'acheteur de ne pas avoir testé adéquatement le marché et de faire assumer par l'entreprise un prix non compétitif. Le troisième risque est celui de l'opportunisme du fournisseur : on soupçonnera le fournisseur de tricher ou de profiter indûment de la dépendance du client, en particulier lorsque le contrat initial a exigé des investissements spécifiques importants (Klein et al., 1978).

Le choix d'un fournisseur incompétent et la possibilité que la conduite future du sous-traitant devienne moralement répréhensible sont pour l'acheteur des sources structurelles d'anxiété. Ces risques ne sont pas vraiment différents aujourd'hui de ce qu'ils étaient hier. Mais, en modifiant le rôle de l'acheteur, l'implantation de l'assurance qualité ne lui permet guère de conserver les méthodes traditionnellement développées pour se prémunir contre ces risques. La familiarité interpersonnelle entre acheteur et fournisseur était autrefois la principale ressource de contrôle des risques. Dans un système plus complexe fondé sur des critères de performance abstraits, il faut apprendre à faire confiance sans connaître personnellement.

\subsection{Traditionnellement courtier: la coordination marchande}

Conformément à l'image la plus répandue de son rôle, l'acheteur cherche traditionnellement à se prémunir contre les risques d'incompétence et d'opportunisme du fournisseur en contrôlant au plus près l'espace et le temps des transactions. Il achète en plus grande quantité et emmagasine plus que nécessaire pour parer aux retards de livraison et au rejet de pièces non conformes. Il préfère la commande répétitive au contrat à long terme pour s'assurer une capacité de réaction et de sanction plus rapide. Il évite de s'approvisionner à une source unique et teste périodiquement le marché des fournisseurs, anciens et nouveaux, qu'il met lui-même en concurrence. Il développe des liens personnalisés avec des fournisseurs habituels escomptant obtenir, au nom de la familiarité et de la confiance ou, dans d'autres cas, sur la base de la domination et de la crainte, une loyauté et un rendement supérieurs à ce que le mécanisme général du marché permet d'espérer (Leenders et al., 1993).

Aussi sincères soient-ils, ces liens personnalisés ne signifient pas que l'acheteur renonce à juger d'après les résultats obtenus dans les contrats antérieurement exécutés et à mettre ses fournisseurs habituels en concurrence périodique. La logique traditionnelle de l'approvisionnement reste toujours, en effet, celle de la commande, c'est-à-dire celle d'un engagement de court terme qui garde toute l'ambiguïté et l'ambivalence d'un contrat conclu avec le sentiment qu'il ne faut jamais se départir d'une méfiance de bon aloi 
(Lamming, 1993). La structure très fermée des documents contractuels accompagnant les appels d'offres et les commandes atteste de la conception «adversariale» plutôt que «partenariale» qui préside aux relations d'échanges établies au coup par coup (Burt, 1989).

L'acheteur peut sans doute prendre ses distances avec cette logique officielle de l'organisation et faire en sorte que le renvoi aux documents contractuels n'ait qu'une incidence superficielle sur le cours de sa pratique. Son autonomie opérationnelle ne cesse pourtant pas de s'exercer dans la logique de la commande, même à l'égard des fournisseurs qui lui sont le plus familiers. L'adoption d'une optique d'engagement à moyen ou long terme, à travers une convention favorisant une définition large et évolutive des obligations des partenaires, supposerait, en effet, que l'acheteur renonce au calcul de type marchand sur lequel se fonde l'essentiel de sa protection contre les risques du métier.

Dans la coordination de type marchand, les risques de non-qualité, de prix non compétitif et d'opportunisme restent, il est vrai, assez élevés. La méfiance de bon aloi fait en sorte que chacun préserve son quant-à-soi, contrôle restrictivement le flux d'informations et garde une distance minimale. Le contrôle du client sur l'exécution des contrats, à supposer qu'on s'en préoccupe, ne peut que rester aléatoire. En contrepartie, les conséquences des risques assumés sont réduites par le court terme de l'engagement et par la valeur modeste ou minimale du contrat. La propension de chacun à protéger sa réputation sur le marché et la familiarité interpersonnelle acquise donnent au surplus une garantie implicite qu'advenant un problème dans l'exécution du contrat, les parties collaboreront efficacement à sa solution. Enfin, la pratique de l'approvisionnement auprès d'un minimum de deux sources donne l'assurance de pouvoir passer d'un fournisseur défaillant à un autre dans un délai assez court.

S'il existe une complicité dans cette coordination marchande, elle est plutôt le fait des individus affectés respectivement à la promotion des ventes et aux achats que celui des entreprises du sous-traitant et du client considérées globalement. Le langage, les sous-entendus, l'ensemble de la communication interfirmes relèvent plus du marketing que de la production (Prus, 1989). Autant les parties se soucient de la détermination expresse des aspects commerciaux du contrat (prix, quantité, délai et lieu de livraison, mode de paiement), autant elles acceptent de s'en remettre aux vagues prescriptions des «règles de l'art» ou à la reconduction implicite de «ce qui s'est fait dans le passé » pour tout ce qui concerne les critères et le contrôle de la production. La référence expresse au devis fourni par le client ne trompe personne, tant il est clair de part et d'autre qu'il ne peut s'agir, dans le meilleur des cas, que 
d'un schéma initial auquel il faudra ajouter bien des précisions avant qu'il devienne opérationnel.

Pour tout ce qui concerne l'exécution du contrat, la logique marchande fait prévaloir une conception essentiellement pragmatique de la coordination interfirmes. L'acheteur fera raisonnablement confiance au fournisseur jusqu'à preuve du contraire. Il manifestera alternativement sa propension à s'engager plus profondément dans une relation de collaboration ou, au contraire, sa préoccupation de maintenir une saine distance d'affaires. Cette ambivalence est inhérente à l'activité du marché qui incite contradictoirement à rechercher des alliances payantes et à rappeler aux partenaires qu'ils ne doivent jamais rien tenir pour définitivement acquis.

L'évaluation de la performance du fournisseur est à l'avenant. L'acheteur y procédera de façon périodique et sans planification précise. Il cherchera moins à mesurer les progrès accomplis par le fournisseur qu'à le noter en fonction des progrès qu'auraient pu faire ses concurrents depuis le dernier appel d'offres (nouveau produit, prix plus bas). En ce qui concerne la qualité, la note de passage lui sera octroyée si aucune plainte n'a été exprimée et si sa performance reste conforme à des «règles de l'art» d'autant plus généralement admises que personne n'assume la responsabilité de leur application ou ne se sent habilité à dire sur quelle moyenne de groupe elles se fondent. Conscient ou non de la part d'arbitraire de cette évaluation en fonction du marché, l'acheteur se réserve toujours la possibilité de conclure à un échec du fournisseur et de le sanctionner par un retour à la case départ. Le cas échéant, le non-renouvellement du contrat marquera un temps d'arrêt forcé et souvent une régression de la coordination interfirmes.

La pratique traditionnelle de l'approvisionnement tend dans une large mesure à laisser l'acheteur se débrouiller seul avec les incertitudes et les contradictions du marché. Les efforts déployés récemment au nom d'une conception plus professionnelle de sa fonction lui procurent de meilleurs outils pour connaître et juger l'évolution du marché. Mais, ils ne sauraient faire disparaître le coefficient aléatoire important de toute décision à prendre et à justifier en fonction des conditions du marché. Puisqu'on lui impute personnellement la responsabilité du choix et du rendement du fournisseur sans lui fournir de critères fiables et universels, il ne faut pas se surprendre de voir l'acheteur calculer ses risques de façon conservatrice et minimale, en misant autant que possible sur la reconduction du passé comme garantie de l'avenir. Aussi longtemps que la logique de l'approvisionnement demeurera celle du marché, l'acheteur cherchera à maximiser les possibilités de répéter les bons résultats obtenus dans le passé plutôt que les chances d'atteindre à l'àvenir de meilleurs résultats. Son évaluation des risques ne se fera en fonction d'un progrès à venir qu'à partir du moment où la coordination 
interfirmes sera globalement conçue dans une optique industrielle plutôt que marchande.

\subsection{De plus en plus entraîneur: la coordination industrielle}

Dans l'optique nouvelle où l'approvisionnement repose sur un travail d'équipe, la collaboration étroite entre les experts de l'assurance qualité et les acheteurs favorise le renforcement des préoccupations de qualité dans les décisions d'achat. En ce qui concerne plus particulièrement les contrats de sous-traitance, il devient clair que l'approvisionnement doit désormais être traité comme une opération de production et non d'échange. En y introduisant des exigences techniques de plus en plus précises, l'implantation de l'assurance qualité soumet le processus de sous-traitance à la logique industrielle d'accroissement de la productivité et suspend, en conséquence, le jugement marchand (Eymard-Duvernay, 1989).

L'impact de ce changement d'optique sur la pratique du métier d'acheteur est bien connu. Il lui faut tendre désormais à conclure des contrats à plus long terme, développer des relations de coopération plus étroites avec les fournisseurs privilégiés de l'entreprise, accepter éventuellement le risque d'un fournisseur unique, négocier des prix et des escomptes qui fassent profiter le client des gains de productivité réalisés par les fournisseurs grâce à l'allongement de la durée des contrats et à la discipline acquise à travers le contrôle systématique de la qualité. Pour chacune de ses décisions, l'acheteur peut et doit compter sur l'assistance des experts locaux de l'assurance qualité et de l'administration de contrat. Il peut légitimement considérer que les défaillances éventuelles des fournisseurs relèveront désormais d'une responsabilité partagée.

La réduction de l'autonomie individuelle de l'acheteur n'est pas le seul prix à payer pour tirer profit du travail en équipe. Sa participation active à un système de coordination industrielle suppose, en effet, qu'il adhère à une conception nouvelle des objectifs à atteindre et fasse confiance à des modes de contrôle sur lesquels il n'a pas une prise directe et encore moins exclusive. L'approche devient plus technocratique que pragmatique. Il ne suffit plus que le fournisseur fasse aussi bien que dans le passé; il faut qu'il réponde de mieux en mieux aux besoins. L'objectif à atteindre n'est pas défini par le niveau de qualité déjà réalisé, mais par un standard abstrait, une projection future, dont le niveau peut être haussé indéfiniment (Soukup, 1987). Dans la logique technocratique, on fait confiance parce qu'on a prévu scientifiquement et en présumant que les échecs eux-mêmes permettront de mieux atteindre un jour les objectifs fixés. L'avenir fera tôt ou tard mentir le passé. 
La recherche d'un rendement toujours croissant incite à évaluer le fournisseur de façon continue, sur une base formative autant que sommative. Il sera désormais noté en fonction de ses progrès, par rapport à une norme abstraite d'excellence plutôt qu'en comparaison avec ses concurrents. La référence aux standards du marché devient de toute façon inadéquate et peu pertinente dès lors que le client consent à son fournisseur des avantages d'exclusivité ou des privilèges grâce auxquels il devrait normalement atteindre un niveau de rendement de plus en plus inaccessible à ses anciens concurrents. Dans la mesure où il développe les habiletés lui permettant de répondre de plus en plus efficacement aux besoins spécifiques de son client, le sous-traitant justifiera le maintien à long terme de sa position privilégiée (Asanuma, 1989).

Pour choisir le bon fournisseur, celui qui prouvera son aptitude à l'amélioration continue de la productivité, et pour convenir avec lui des conditions contractuelles favorisant l'atteinte des nouveaux objectifs, l'acheteur doit s'en remettre aux modes de contrôle et aux ressources du système de l'assurance qualité. Il devra d'abord faire confiance à l'autocontrôle du fournisseur, présumer que ce dernier saura développer une expertise autonome, qu'il assumera une responsabilité croissante et restera fidèle aux prescriptions du contrôle de qualité en dépit du fait que la surveillance du client tendra à se faire distante et indirecte (Sako, 1992).

L'acheteur devra se fier, en deuxième lieu, au contrôle réalisé par des tiers experts de l'assurance qualité opérant à distance et selon des standards extra-locaux. À cet égard, Alcan s'est initialement référée au QMI, une division de l'ACNOR dont le siège social est situé à Mississauga et qui a un bureau d'affaires à Pointe-Claire au Québec. Plus récemment, Alcan a décidé de reconnaître aussi les certificats d'assurance qualité délivrés par les autres organismes accrédités par le Conseil canadien des normes. L'acheteur est ainsi invité à faire confiance à la réputation de ces organismes externes offrant leurs services de contrôle à tous ceux qui doivent faire confiance à des agents qu'ils ne connaissent pas personnellement (Shapiro, 1987). L'acheteur devra se fier à la qualité des processus de certification offerts et au sérieux des audits annuels censés attester du maintien des standards de contrôle de la qualité chez les fournisseurs certifiés.

Pour que l'autocontrôle du fournisseur et les rapports d'évaluation de l'organisme de certification soient crédibles à ses yeux et influencent réellement ses décisions, l'acheteur devra finalement apprendre à faire confiance aux documents écrits beaucoup plus qu'il ne l'a fait traditionnellement. Avec le passage de la coordination marchande à la coordination industrielle, la communication écrite change, en effet, de fonction. L'écrit remplissait traditionnellement une fonction probatoire. Il témoignait, mieux et plus fidèlement que la mémoire orale, des conventions de départ et des 
changements apportés en cours d'exécution du contrat. Il scellait les décisions et protégeait les parties concernées contre les «trous de mémoire», les tentatives de réinterprétation, les changements de personnel.

L'assurance qualité tend à augmenter le volume et la circulation de la documentation écrite. Elle fait surtout en sorte que l'écrit serve désormais de plus en plus de point de repère et de base d'amélioration du processus de production. Dans la sphère des opérations, auparavant le lieu privilégié des échanges verbaux parce qu'il s'agissait d'abord de faire plutôt que de dire les choses, la documentation écrite rendra compte, aux fins des décisions futures, des résultats mesurés à intervalles réguliers. La détermination écrite des exigences techniques initiales, les rapports de performances atteintes à chacune des étapes d'exécution et la mise à jour constante des devis et des modèles utilisés permettront de constituer pour chaque produit un dossier ou un historique de production permettant une évaluation a posteriori du chemin parcouru et une détection plus facile des sources d'erreurs ou de contreperformances. L'écrit devient, en somme, un instrument majeur d'introduction de l'esprit de discipline et de méthode indispensable à la coordination industrielle. Il fournit la base de la réflexivité précise et ininterrompue qui caractérise tous les systèmes-experts (Giddens, 1990).

Le dossier de production n'est pas une simple source additionnelle d'informations consultée par l'acheteur au moment où il doit décider du renouvellement ou non d'un contrat. Il est censé devenir un instrument de travail couramment utilisé dans l'exercice d'un rôle nouveau qui consiste à suivre l'évolution de chaque fournisseur et à orienter son développement dans le sens des aptitudes acquises et des besoins du client. L'acheteur ne peut plus se limiter à agir comme un simple courtier prospectant le marché pour établir des liens contractuels avantageux pour ses clients. De concert avec les experts de l'assurance qualité et les usagers, il devient pour ainsi dire l'entraîneur d'une équipe de fournisseurs réguliers qu'il doit guider sur la voie d'un partenariat dont chaque étape correspond à un degré d'intensité plus élevé de la coordination interfirmes.

À travers des décisions d'achat de plus en plus interdépendantes, l'acheteur gère la division du travail entre ses fournisseurs, sur une base géographique, en tenant compte des avantages de la proximité du fournisseur et de l'établissement à servir et, d'un point de vue fonctionnel, en répartissant les créneaux de production selon la compétence acquise par chaque fournisseur ou celle que le client souhaite lui voir acquérir compte tenu de ses besoins. Les fournisseurs se trouvent ainsi assignés à des aires géographiques (locale, régionale, nationale, voire internationale) et à des spécialités (fabrication ou installation; soudure, mécanique ou coulage ; pièces de grandes, moyennes ou 
petites dimensions, etc.) qui deviennent autant de «marchés internes» dispensant le client de recourir au marché général (Favereau, 1989).

Les fournisseurs souscriront d'autant mieux à la division du travail induite par les décisions de l'acheteur si ce dernier peut leur assurer un volume d'achats significatif et maintenir l'incitation aux gains de productivité par des mécanismes incitatifs appropriés. Cela suppose une autonomie et des attributions nouvelles pour l'exercice de son rôle. L'acheteur doit pouvoir regrouper les achats des établissements de l'entreprise, consolider les contrats de sous-traitance en fonction de la connexité des opérations de production et des compétences requises, réunir les ressources techniques, humaines et éventuellement financières que le client mettra à la disposition du fournisseur. Acteur clé d'une coordination horizontale avec les sous-traitants (Aoki, 1991), l'acheteur ne sera un entraîneur efficace qu'à la condition de jouir d'une confiance plus étendue au sein de son organisation. Sans cette confiance, l'arrimage du fonctionnement interne de l'entreprise cliente avec celui de l'entreprise sous-traitante n'atteindra pas le degré de cohésion requis pour que le pari sur l'avenir, sur l'amélioration continue de la qualité et sur le partenariat avec les fournisseurs soit tenu. À défaut d'un tel arrimage, il est peu probable, en effet, que le sous-traitant fasse confiance au système d'assurance qualité au point de modifier significativement son évaluation des risques.

\section{Les risques du sous-traitant}

Dans la pratique de la sous-traitance industrielle, deux risques notoires sont des sources de préoccupation constante pour l'entrepreneur : gagner un contrat déficitaire à la faveur d'une soumission trop basse et perdre un contrat rentable au profit d'un concurrent. L'implantation de l'assurance qualité ne fait pas disparaître ces risques. Elle en modifie cependant les possibilités d'occurrence et les conséquences.

\subsection{La soumission trop basse}

Dans la coordination marchande traditionnelle, la discontinuité et le quant-àsoi qui marquent les rapports interfirmes accroissent les possibilités que le sous-traitant sous-estime les coûts du contrat, ses difficultés d'exécution ou la rigueur des exigences du chargé de projet désigné par le client. S'il gagne un contrat déficitaire, le sous-traitant sera contraint de choisir entre des alternatives qui comportent toutes des conséquences négatives à court ou à moyen terme. Il peut s'en tenir intégralement à ses engagements et absorber la perte financière à même les profits des autres contrats ou sa marge de crédit. Il peut réclamer des suppléments de coûts, mais entachera alors sa crédibilité auprès 
du client. Il peut modifier son mode de fonctionnement interne et faire absorber la perte par ses employés, directement ou indirectement. Il peut, enfin, réaliser des économies en jouant sur les imprécisions de l'appel d'offres et le flou des règles de l'art au risque de fournir un produit de mauvaise qualité.

Parce que la soumission trop basse est susceptible d'entraîner des conséquences négatives pour le client, l'acheteur cherche normalement à prévenir les calculs erronés des sous-traitants et non à conclure hâtivement un marché de dupes. Il dispose à cet égard de certains moyens de contrôle à l'intérieur d'une marge de manœuvre qui reste toutefois assez étroite. Premièrement, il n'invitera pas n'importe qui à soumissionner. Mais, on s'attend à ce que ses appels d'offres s'ouvrent régulièrement à la possibilité de voir émerger le jeune loup capable de relancer la concurrence chez les sous-traitants et pouvant légitimement réclamer la chance de se mettre en valeur. Deuxièmement, l'acheteur pourra faire observer au plus bas soumissionnaire un écart troublant dans l'estimé des coûts. Mais, il risque ainsi de manquer ou de paraître manquer à l'éthique de la procédure d'appel d'offres, ce qui le rend vulnérable aux récriminations des compétiteurs. Enfin, pour encourager l'entrepreneur à assumer les conséquences d'un contrat déficitaire, l'acheteur peut lui faire entrevoir un profit compensateur sur un contrat subséquent. Mais, il s'agit là, ou bien d'une hypothèque très embarrassante pour l'acheteur, ou bien d'une perspective d'avenir trop incertaine pour le sous-traitant.

Dans ces conditions, c'est d'abord au sous-traitant lui-même qu'incombe la responsabilité de contrôler le risque de la soumission trop basse et de décider s'il répondra à l'appel d'offres «en jouant sûr ou en faisant le paon ». Il lui est loisible d'envisager la soumission de façon stratégique en calculant ses coûts au plus bas lorsque son objectif premier est de mettre le pied ou de revenir dans le cercle des fournisseurs du client. Le déficit du contrat se conçoit alors comme l'investissement requis pour acquérir ou récupérer auprès du client le statut minimal permettant d'espérer des profits ultérieurs.

Une fois ce statut acquis ou récupéré, le sous-traitant calculera ses coûts à la lumière de son expérience professionnelle générale et de sa connaissance plus particulière du client. S'il a déjà été lui-même à l'emploi du client ou d'un de ses fournisseurs, s'il connaît personnellement le chargé de projet ou les usagers impliqués, s'il a établi des rapports de confiance avec différentes personnes au sein de l'organisation cliente, s'il a déjà réalisé des contrats pour le client, le sous-traitant disposera normalement d'informations multiples réduisant l'incertitude des coûts à prévoir. Lorsque la complexité technique du contrat est grande et que les exigences du client s'annoncent rigoureuses, il peut se ménager un coussin pour protéger son profit en escomptant raisonnablement que ses concurrents feront de même. 
Quoique les moyens de protection dont disposent l'acheteur et le soustraitant ne soient pas négligeables, le risque de la soumission trop basse reste généralement plus grand dans un régime de coordination marchande. Dans une coordination du type industriel, le contrôle de qualité et l'amélioration des procédés de gestion permettent au sous-traitant de mieux connaître ses coûts de production. Les exigences et les difficultés d'exécution du contrat sont mieux documentées. La concentration des communications interfirmes au niveau des responsables du contrôle de qualité réduit les perturbations dues à l'intervention de personnes trop nombreuses ou peu familières avec l'évolution de la relation entre les deux entreprises.

La communication continue entre les représentants des parties dans le cadre de rapports contractuels d'une durée plus longue a surtout pour effet de désacraliser la soumission. Elle n'est plus le grand moment décisif où doivent être fixés de façon intangible tous les termes du contrat. La production d'un bien industriel devant répondre de plus en plus spécifiquement au besoin du client requiert un mécanisme de régulation plus séquentiel et plus ouvert à des adaptations fréquentes (Williamson, 1979; 1985). L'ancienne technique contractuelle, coulée dans le moule formaliste de l'appel d'offres, se prête mal à cette régulation souple et évolutive. La soumission n'est plus la bible du rapport marchand d'autrefois. Elle devient un point de repère parmi d'autres, une occasion de dresser un bilan et de convenir des adaptations requises pour la poursuite de la relation.

\subsection{La perte d'un contrat rentable}

Si l'obtention d'un contrat à la faveur d'une soumission trop basse crée au sous-traitant un véritable casse-tête, il n'est pas exagéré de considérer la perte d'un contrat rentable comme le début d'une crise organisationnelle dont l'ampleur variera selon la valeur économique du contrat perdu. Du coup, une partie de l'organisation constituée par le sous-traitant devient inutilisée, sinon encombrante. À défaut d'une solution rapidement trouvée, il faudra envisager de se départir d'un équipement de production et d'un personnel spécialisés au risque de ne plus les trouver disponibles plus tard.

La recherche d'une solution de rechange fait elle-même partie du problème puisqu'elle suppose une ambivalence stratégique productrice d'insécurité : faut-il changer le moins possible et rester en position de regagner le contrat perdu ou, au contraire, se tourner résolument vers un autre créneau d'activités ? La perte d'un contrat significatif provoque à cet égard une crise de confiance aux dimensions multiples. Le sous-traitant a-t-il entretenu des illusions sur la qualité de son travail et de son expertise ? Était-ce naïf de sa part de faire confiance à l'attachement du client et de ses représentants 
envers leur relation mutuelle ? Peut-il croire encore en la justice du système d'adjudication des contrats du donneur d'ordre ? La perte du contrat entacherat-elle la crédibilité de l'entreprise au sein du marché?

Le contrôle que peut exercer l'entrepreneur eu égard à la perte d'un contrat rentable et les conséquences négatives qui en découleront ne sont pas les mêmes selon que les relations d'affaires concernées relèvent d'une coordination marchande ou industrielle. Dans le régime traditionnel de soustraitance, le contrôle de l'entrepreneur est plutôt faible, mais la crise provoquée par la perte du contrat sera généralement moins grave. Dans un régime de coordination intensifiée par l'implantation d'un système d'assurance qualité, l'entrepreneur acquiert normalement une meilleure protection contre ce risque ; mais si son entreprise en vient à perdre un contrat avec le client, les conséquences seront probablement beaucoup plus grandes. La perte d'un contrat pourra fort bien signifier la perte d'une relation dans laquelle le soustraitant a beaucoup investi au point d'y engager son identité même. Avec le passage à la coordination industrielle, le risque est devenu typiquement moderne, c'est-à-dire moins probable, mais plus grave. Il vaut la peine de s'arrêter plus longuement à ces différences, car elles permettront de mieux comprendre en quoi le défi de l'assurance qualité réside dans l'acceptation par l'entrepreneur d'un risque nouveau.

\subsubsection{Dans la sous-traitance traditionnelle}

Dans la sous-traitance traditionnelle, la forte probabilité de la perte d'un contrat rentable et l'ampleur limitée de ses conséquences tiennent au fait que la coordination interfirmes se réalise dans un temps et un espace réduits, généralement à la faveur des rapports personnalisés qu'entretiennent les acteurs établis à faible distance physique les uns des autres. Compte tenu de la logique marchande qui préside aux relations interfirmes, les représentants de l'entreprise cliente et du sous-traitant savent d'expérience que le fournisseur même le plus habile à soigner ses relations est condamné à devoir recommencer périodiquement un cycle d'échanges où la perte d'un contrat acquis de longue date donne le signal d'un retour à la case départ.

La personnalisation des rapports entre les acteurs impliqués est paradoxalement un facteur non négligeable de la perte d'un contrat aussi bien qu'une ressource favorisant l'obtention d'un premier contrat. Même s'il fournit un bon rendement qualité/prix, le sous-traitant peut perdre le contrat parce qu'un changement de personnel s'est produit dans l'entreprise cliente au niveau des usagers ou du service d'approvisionnement ou parce que la durée même de la relation d'affaires servira d'argument à tous ceux qui ont intérêt à ce qu'on soupçonne l'acheteur de favoritisme. 
La proximité physique des entreprises sur le marché local de la soustraitance favorise de la même façon la perte aussi bien que l'obtention d'un contrat. Elle fournit au client des ouvertures d'autant plus accessibles que le critère de rendement est celui du marché environnant plutôt que celui du niveau atteint par le sous-traitant le plus expérimenté. Au sein de ce marché, tous savent bien que l'acheteur sera tôt ou tard forcé de tester réellement la concurrence entre les fournisseurs et d'appliquer ensuite la sanction qui fera passer un contrat du sous-traitant le plus expérimenté à celui qui s'est montré le plus offrant. L'acheteur peut d'autant moins résister à la logique marchande qu'il ne dispose pas des données techniques cumulatives qui lui permettraient de démontrer aux gestionnaires de l'entreprise cliente le niveau de performance réellement atteint par le sous-traitant actuel.

Dans ces conditions, les sous-traitants adoptent une règle de prudence qui consiste à ne pas trop s'éloigner du niveau de compétence généralement atteint par les entreprises du marché local et à ne pas s'engager trop profondément dans une relation d'affaires toujours susceptible de connaître une suspension abrupte. Les exigences techniques du contrat à exécuter et l'espoir d'un renouvellement de contrat à moyen et à long terme les amènent sans doute à investir dans une nouvelle relation en recherchant une coordination accrue avec l'entreprise du donneur d'ordre. Cette propension sera d'autant plus forte si les achats du client représentent une part majeure du volume d'affaires du sous-traitant. Même dans ce cas, la logique marchande incite toutefois à ne pas dépasser le seuil au-delà duquel une plus grande coordination devient incompatible avec le maintien de solutions de rechange sur lesquelles on pourra se replier advenant la rupture de la relation.

\subsubsection{Dans un régime d'assurance qualité}

L'implantation d'un système d'assurance qualité suppose que les partenaires de la sous-traitance industrielle s'engagent dans un effort de coordination qui les mène à investir au-delà du seuil que la logique marchande inciterait à ne pas dépasser. La référence aux normes abstraites et décontextualisées de l'assurance qualité contient la promesse d'une coordination interfirmes moins sujette aux aléas des rapports interpersonnels et de la concurrence locale. Les sous-traitants peuvent considérer que le risque de perdre un contrat rentable dans lequel ils auraient investi au-delà de la règle de prudence traditionnelle sera désormais moins grand.

Il y aura d'abord moins d'appels d'offres et, par conséquent, moins d'occasions de soumissionner pour les concurrents. La tendance sera de négocier le renouvellement des contrats avec les sous-traitants actuels plutôt que de les mettre systématiquement en concurrence avec leurs anciens 
compétiteurs. La constitution d'un groupe de fournisseurs privilégiés aura normalement pour effet de libérer l'acheteur des pressions visant à faire une place aux jeunes loups. Dans un régime d'assurance qualité, le fournisseur certifié détenant le contrat ne sera normalement mis en concurrence qu'avec d'autres fournisseurs certifiés.

L'intensification de la coordination industrielle entre le client et ses principaux sous-traitants crée ainsi une distance croissante entre le client et les petits sous-traitants du marché. Les exigences techniques à satisfaire et les responsabilités à assumer deviennent de plus en plus hors de portée des nouveaux venus sur le marché. Les sous-traitants principaux sont incités, directement ou indirectement, à jouer à cet égard un rôle de médiation en prenant eux-mêmes le contrôle des petites entreprises reléguées au statut de fournisseurs occasionnels et promises de plus en plus à une sous-sous-traitance gérée par les fournisseurs principaux. Le rehaussement des exigences de qualité entraîne une stratification plus rigide dans le marché de la soustraitance.

En outre, le risque de perdre un contrat rentable diminue parce que les sous-traitants privilégiés sont moins susceptibles de pratiquer la soumission purement stratégique. Chaque sous-traitant a développé une organisation plus complexe qu'il n'a pas le loisir de chambarder pour obtenir un nouveau contrat. L'impulsion donnée à l'entreprise pousse désormais à aller de l'avant en maintenant les standards de qualité et de prix. La perspective de brusques retours en arrière pour travailler à meilleur compte en rabaissant le niveau de qualité apparaît au sous-traitant comme une incohérence organisationnelle de plus en plus démotivante. Il cesse d'être systématiquement à l'affût de tout nouveau contrat et se concentre dans l'aire de spécialisation que tend à lui réserver la segmentation du marché découlant des nouvelles politiques d'approvisionnement du client.

Il n'est pas dit que les sous-traitants souscriront d'emblée à la nouvelle organisation industrielle favorisée par leur client et n'entretiendront aucune velléité d'en sortir. Des tensions, voire des conflits ouverts, surgiront chaque fois que les décisions des acheteurs ou les caprices de la conjoncture économique créeront des disparités dans le rendement et la sécurité offerts par les différents créneaux de spécialisation. Aussi longtemps que le client disposera d'un pouvoir de marché important, ses principaux sous-traitants seront toutefois amenés à concevoir leurs rapports de concurrence et de coopération dans l'optique particulière de l'organisation de la production requise pour satisfaire aux besoins du client, plutôt que dans l'optique générale qui prévalait à l'époque où le marché local répondait à une demande beaucoup moins articulée du client. 
L'intensification de la coordination entre le donneur d'ordre et chacun de ses sous-traitants privilégiés diminue le risque d'une rupture de leur relation d'affaires dans la mesure même où elle accroît leur interdépendance. Pour augmenter le rendement des contrats de sous-traitance, les parties ont dû manifester plus d'ouverture à l'autre, un esprit de coopération plutôt que de méfiance, une communication plus franche, un souci plus grand de constituer un dossier technique fiable pour chaque produit fourni, une volonté plus nette de planifier l'avenir en commun. L'organisation de la production s'est faite de plus en plus dans l'optique d'un espace industriel qui établit des liens directs entre les processus ayant cours au sein de chacune des deux entreprises en minimisant autant que possible l'effet des frontières organisationnelles. Les objectifs et les moyens ont été déterminés sur la base des possibilités et des contraintes techniques sans qu'on se reconnaisse limité par la durée formelle et la stricte logique des termes du contrat en vigueur. Plus étendue dans l'espace et dans le temps, la coordination interfirmes est devenue telle que les partenaires se trouvent désormais «enfermés dans un monopole bilatéral » (Williamson, 1979; Lorenz, 1988; Schonberger, 1982) dont ils ne pourront se dégager qu'au prix d'une crise organisationnelle profonde.

Moins probable dans ces conditions, la rupture d'un contrat de soustraitance comporte en contrepartie des conséquences beaucoup plus lourdes. Elle signifie pour l'entrepreneur la perte des profits générés par une expertise poussée et spécifique qu'il aura beaucoup de mal à reconvertir pour d'autres clients, du moins sur le marché local. La firme sous-traitante connaît à cet égard un problème similaire à celui du chômage de l'ouvrier spécialisé qui a lié son sort à celui de son employeur. Comme l'ouvrier spécialisé, le fournisseur privilégié peut cependant compter sur une protection supérieure à celle de l'occasionnel. Puisque les risques du fournisseur et de son client sont devenus étroitement interreliés, on peut s'attendre à ce que l'échec du premier soit ressenti par le second comme une défaillance de son propre système de gestion et de ses calculs stratégiques. On ne s'étonnera donc pas de voir le client assumer une partie du problème de son sous-traitant, lui permettre de corriger sa défaillance et collaborer activement à la recherche d'une solution pour l'avenir. La rupture d'une relation où la coordination fut intense n'impose pas seulement au client de recommencer avec une autre firme un processus d'évolution toujours laborieux, elle peut fort bien provoquer une crise de confiance dans les vertus du système d'approvisionnement mis en place par le donneur d'ordre et dans la pertinence de sa stratégie d'organisation du marché.

Si l'intensification de la coordination interfirmes à la faveur d'un programme d'assurance qualité protège mieux le sous-traitant contre le risque de perdre un contrat rentable, elle n'équivaut toutefois jamais à une garantie de maintien d'une relation structurante pour chacun des partenaires. Moins 
encore que l'ouvrier spécialisé, le sous-traitant ne pourra jamais prétendre à une sécurité de contrat absolue. Le régime d'assurance qualité et la perspective du partenariat placent ainsi l'entrepreneur devant un dilemme. Pour obtenir des contrats profitables avec le donneur d'ordre, il lui faudra accepter dorénavant de s'engager dans un processus de coordination beaucoup plus exigeant en liant son sort à celui de l'entreprise cliente (Leavy, 1994). Accédant ainsi à un nouveau statut au sein du marché, il lui sera plus difficile de maintenir sa capacité de concurrencer efficacement ses anciens compétiteurs et de se reconvertir sur cette base advenant la rupture de la relation (Podolny, 1993).

Une solution logique qui permet de s'engager à fond dans une relation favorable à un accroissement de l'expertise du sous-traitant et d'éviter en même temps une trop grande dépendance envers le donneur d'ordre est de concevoir l'avenir de l'entreprise en fonction de clients plus exigeants que ne le sont ceux du marché local, préférablement dans le créneau d'activités où se retrouvent des clients ayant des besoins semblables à ceux du donneur d'ordre (Houssiaux, 1957). Les entrepreneurs rencontrés dans le cadre de notre étude font eux-mêmes un lien étroit entre la certification à l'assurance qualité et leur propension à s'inscrire de plus en plus dans la dynamique d'un marché national et nord-américain, notamment celui de la sous-traitance auprès des nouvelles alumineries du Québec. La logique marchande de l'approvisionnement traditionnel incitait 1'acheteur à soutenir plusieurs fournisseurs d'expertises comparables; la logique industrielle de l'assurance qualité incite l'entrepreneur à rechercher plusieurs clients aux besoins et aux exigences comparables.

Le caractère éminemment moderne du défi posé par l'assurance qualité apparaît encore ici de façon très claire. Les sous-traitants sont invités à lier leur sort à une stratégie de développement qui s'appuie sur la compétence qu'on prévoit acquérir dans le futur plutôt que sur celle qu'on a déjà acquise et sur la demande potentielle d'un marché élargi plutôt que sur les possibilités du marché local. On ne doit pas s'attendre à ce que les sous-traitants acquièrent instantanément une telle confiance en l'avenir et s'engagent dans cette voie autrement que d'une façon graduelle. Une difficulté majeure réside à cet égard dans le caractère contradictoire des messages émis par le donneur d'ordre au stade de l'implantation du système d'assurance qualité.

\section{Les ambivalences du partenariat}

Les entrepreneurs rencontrés dans le cadre de notre recherche n'ont pas manqué de souligner ce qu'ils ont perçu comme des incohérences dans le processus d'implantation de l'assurance qualité enclenché par Alcan. L'élagage du marché au nom du contrôle de qualité n'aurait pas été aussi net 
qu'on l'avait annoncé. Le client n'aurait pas osé appliquer dans toute sa rigueur la règle de la certification comme condition indispensable pour

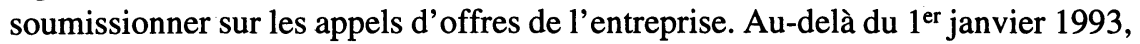
des fournisseurs non encore certifiés auraient été invités à soumissionner. Des appels d'offres auraient été retardés pour permettre à certains fournisseurs d'obtenir leur certification dans des conditions qui risquaient de faire du certificat d'assurance qualité une bannière plus révélatrice d'une habile stratégie de marketing que d'une maîtrise solide des contrôles de qualité.

Les sous-traitants qui avaient obtenu leur certification dans le délai fixé se sont également montrés déçus par la teneur des discussions avec le client au moment de négocier le renouvellement des contrats. Malgré les principes de l'assurance qualité, les considérations de prix l'emporteraient souvent sur les questions de qualité. L'obsession du prix chez le client amènerait les fournisseurs certifiés à craindre comme avant la concurrence des petites entreprises capables d'offrir des coûts imbattables au détriment de la qualité.

Ceux qui avaient espéré que la mise en place de l'assurance qualité ouvrirait une ère nouvelle où l'on discuterait des moyens d'intensifier la coordination interfirmes et d'améliorer la qualité à la faveur d'investissements communs, avec l'assurance d'un partenariat à long terme, en sont quittes pour une bonne dose de frustration. Le discours du client avait fait naître chez eux des attentes précises, ou à tout le moins un espoir raisonnable, de pouvoir s'affranchir de l'éternel recommencement auquel la logique marchande confine le sous-traitant. L'écart perçu entre le discours et les décisions concrètes les incite maintenant à se demander si l'assurance qualité ne sert pas de prétexte pour permettre au client d'obtenir plus dans ses négociations avec les fournisseurs sans renoncer à recourir périodiquement aux services des jeunes loups. L'expérience récente enseignerait à tout le moins qu'une mise entre parenthèses des principes par le donneur d'ordre est toujours possible (Ramsay, 1990) et que le "partenariat à l'intérieur de la domination» (Leborgne et Lipietz, 1988) se pratique forcément dans l'ambivalence. Comme le montre bien l'organisation industrielle japonaise, le modèle partenarial d'approvisionnement réserve au donneur d'ordre une influence prépondérante sur l'orientation de la coopération interfirmes. Les stratégies de certification des fournisseurs et d'intensification de la coopération n'impliquent pas en elles-mêmes le passage à une collaboration égalitaire (Lamming, 1993).

Des raisons conjoncturelles expliquent certainement en partie les incohérences perçues par les sous-traitants. L'implantation de l'assurance qualité a été préparée au moment où l'entreprise Alcan connaissait des profits records (1987-1990), mais sa mise en œuvre effective a coïncidé avec le début de la récession de l'économie nord-américaine et surtout avec une chute 
considérable du prix de l'aluminium à la faveur d'un marché mondial où l'offre a excédé de beaucoup la demande. Le ralentissement de la production d'aluminium, le repli sur l'entretien des équipements de production plutôt que sur leur remplacement ou sur les projets de construction, la recherche systématique des économies dans l'ensemble des opérations de l'entreprise cliente ont eu pour effet de réduire significativement le volume des achats et de soumettre les acheteurs à des contraintes budgétaires accentuées. Assurance qualité ou non, une telle conjoncture ne peut qu'affecter négativement le volume de la sous-traitance et augmenter la part de la méfiance dans la communication interfirmes.

Il va de soi également que l'implantation de l'assurance qualité constitue une phase de transition entre les modes marchand et industriel de la coordination interfirmes. Cette transition peut difficilement s'effectuer au même rythme pour tous les fournisseurs offrant le potentiel recherché par le client. Au risque de subir la critique des fabricants déjà certifiés, les responsables de l'assurance qualité chez Alcan ont ainsi préféré, dans certains cas, ne pas appliquer

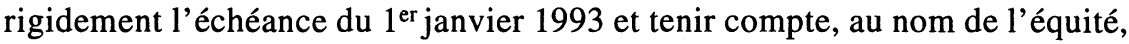
des bons états de service de certains sous-traitants sur le point d'obtenir leur certification. En plus de donner lieu à de tels accommodements, la phase d'implantation de l'assurance qualité est aussi, pour les représentants du client comme pour le personnel des entreprises sous-traitantes, une période d'apprentissage qui n'exclut ni les tâtonnements ni les erreurs. Les sous-traitants sont d'ailleurs parfaitement conscients du fait que la stratégie d'assurance qualité rencontre encore au sein de l'entreprise de leur client diverses résistances qui sont autant de sources potentielles d'incohérences.

La phase d'implantation est, par ailleurs, celle pendant laquelle les parties impliquées cherchent à comprendre les finalités du système d'assurance qualité dans l'optique la plus favorable à leurs intérêts respectifs. Les mots employés pour annoncer une orientation nouvelle ont dans ce contexte une importance stratégique à double tranchant. L'évocation de «partenariat» et de «l'amélioration continue de la qualité » dans le discours du client donne le signal d'une volonté très nette de rompre avec les pratiques traditionnelles de la sous-traitance. Elle fournit par la même occasion des arguments aux soustraitants intéressés par la perspective d'une collaboration de longue durée qui les protégerait des calculs comptables de court terme et leur permettrait de raffiner leur production avec l'assurance sereine d'une continuité de la demande.

Mais la finalité du système ne pouvant être celle d'une amélioration de la qualité sans égard au prix, le client prendra tôt ou tard des décisions dont l'effet, sinon l'objectif, sera de ramener à la baisse les attentes inflationnistes que son discours a fait naître chez ses sous-traitants. Si le donneur d'ordre 
entend favoriser à l'avenir une augmentation de la qualité des produits achetés, il ne renonce pas pour autant à l'avantage comparatif traditionnel de la soustraitance, celui de pouvoir compter sur des coûts de main-d'œuvre et des prix de revient inférieurs aux siens. De même, le régime du partenariat ne signifie pas que le client renonce aux avantages de la concurrence. Il participe plutôt d'une logique de gestion ouverte de la concurrence au sein du réseau des fournisseurs en vue d'un progrès de la productivité générale dont le donneur d'ordre sera le premier bénéficiaire (Itami et Senbongi, 1992). Au terme du processus d'implantation, il sera clair pour tous que la finalité du système est l'amélioration continue du rendement qualité/prix et non de la qualité seule. L'évocation des mots cédera alors la place à la négociation des mesures concrètes susceptibles de maintenir chez chacun des partenaires la motivation à intensifier la coordination interfirmes.

Il faudra alors s'attaquer à des sources plus structurelles d'incohérence ou d'ambivalence. Une question primordiale est, à cet égard, celle de savoir dans quelle mesure la stratégie de partenariat avec des fournisseurs privilégiés implique que le client renonce lui-même à la fabrication. S'il préfère pratiquer la politique dite de «la zone grise» (Nollet et Leenders, 1991), quelles seront les règles d'arbitrage entre les intérêts des employés du client et ceux des fournisseurs? Alcan a suscité beaucoup d'appréhensions chez ses soustraitants en confiant à son groupe d'assurance qualité le mandat d'assister l'atelier d'usinage de l'entreprise dans son processus de certification à l'assurance qualité. Les craintes des sous-traitants à l'égard de cette concurrence interne sont d'autant plus vives qu'ils n'ignorent pas que cette politique du donneur d'ordre répond à de fortes pressions syndicales visant à contrer le recours à la sous-traitance dans un contexte de diminution de l'emploi au sein des établissements d'Alcan. Le client qui ne souhaite pas ou ne peut pas choisir nettement entre les options de faire et faire-faire s'impose la tâche délicate de convaincre ses fournisseurs que son offre d'intégration plus poussée exprime un engagement à long terme plutôt qu'une simple stratégie cyclique.

En partie liée à cette première question est celle de savoir si le client pourra alimenter suffisamment son fournisseur privilégié et lui éviter le démantèlement de son organisation au gré des variations de la demande? Quelles compensations le client peut-il offrir pour les pertes de volume? Si le client s'approvisionne parallèlement auprès d'un deuxième fournisseur privilégié pour éviter la situation de «monopole bilatéral», comment s'effectuera l'allocation des contrats entre les deux fournisseurs principaux (Asanuma, 1989) ? En outre, quelles primes au rendement ou quels autres avantages incitatifs seront offerts au fournisseur privilégié pour que l'accroissement de sa productivité se traduise par une augmentation de la profitabilité 
de son entreprise ? Comment seront répartis entre le donneur d'ordre et ses principaux sous-traitants les risques contractuels et les bénéfices ou «quasirentes relationnelles » découlant de l'intensification de la coopération interfirmes (Aoki, 1991)?

D'autres questions d'ordre structurel ont trait au degré de systématisation du nouveau régime de sous-traitance. Un système d'assurance qualité peut-il fonctionner à deux ou trois vitesses, c'est-à-dire sur la base d'exigences de qualité plus ou moins élevées selon les produits, selon les fournisseurs, ou les deux? Le sous-traitant à qui est confiée la responsabilité d'un groupe de produits réussira-t-il à motiver ses employés à produire une qualité accrue dans certains cas et une qualité moindre dans d'autres cas? La finalité du système pourra-t-elle être atteinte sans que les nouveaux standards de qualité soient imposés aux sous-sous-traitants?

Enfin, l'intensification de la coordination interfirmes en ce qui a trait aux activités de production ne saurait être poursuivie longtemps sans que la coopération doive aussi s'intensifier à d'autres égards. On pense notamment à la question des chevauchements d'activités (Matthews, 1993) et à l'amélioration des modalités de paiement des contrats (Burt, 1989). Les experts de l'assurance qualité pourront-ils amener les gestionnaires de l'entreprise cliente à consentir aux fournisseurs privilégiés des conditions financières inconcevables dans la logique marchande traditionnelle, mais utiles, voire indispensables, dans un régime de coordination plus intense?

\section{Conclusion}

Parce qu'elle donne à la coordination interfirmes une extension plus grande dans le temps et dans l'espace, l'assurance qualité impose à l'acheteur et au sous-traitant d'évaluer différemment les exigences de leur métier et d'assumer des risques nouveaux. En dépit des incohérences qui l'affectent, particulièrement au stade de l'implantation, le système d'assurance qualité leur offre, en contrepartie, la possibilité d'échapper aux risques anciens et la promesse de pouvoir compter sur des ressources accrues pour éviter les conséquences négatives des nouvelles façons de faire. Outre l'assistance technique de ses experts locaux, le système promet à l'acheteur le soutien d'une équipe partageant avec lui la responsabilité du rendement des fournisseurs. Il procure au sous-traitant l'interdépendance accrue qui lui permettra de négocier plus facilement le renouvellement de ses contrats. Le fonctionnement du système d'assurance qualité dans la sous-traitance industrielle suppose, en somme, que le donneur d'ordre gère l'organisation interne de son entreprise en considérant que les fournisseurs privilégiés en font virtuellement partie, même s'ils n'en sont pas membres à part entière (Magnet, 1994). Pour la PME sous-traitante, 
la condition de fournisseur privilégié implique la gestion du risque stratégique lié à sa dépendance envers la grande firme (Julien et Marchesnay, 1988).

Au-delà des contraintes et des risques que les acteurs devront apprendre à assumer, le défi de l'assurance qualité réside aussi dans la régulation des risques du système lui-même. Bien qu'on devance ici l'expérience concrète qui a alimenté notre réflexion, on peut d'ores et déjà prévoir que le système d'assurance qualité sera confronté tôt ou tard aux aléas communs à tous les systèmes-experts.

Il y a d'abord le risque technique d'une sophistication ou trop grande ou trop faible du système mis en place. Rien ne garantit que les exigences imposées par le système ne dépasseront pas un jour le niveau de rendement optimal que peut atteindre la coordination interfirmes dans un contexte donné (Ravix, 1990). En sens contraire, on ne peut tenir pour acquis que les experts sauront toujours répondre aux incertitudes de l'environnement en mettant au point les mécanismes d'ordre qui seraient nécessaires (Luhmann, 1979; Giddens, 1990). Il y a ensuite le risque social d'une technologie qui perturbe les rapports sociaux anciens en imposant des contraintes auxquelles les acteurs ne pourront s'adapter durablement ou qu'ils intégreront de manière dysfonctionnelle. Si, comme le prétend la publicité de l'entreprise Xerox (1993), l'assurance qualité est une course où il n'y a pas de ligne d'arrivée, il faut prévoir que la fatigue des participants dépassera éventuellement la limite du tolérable. Les acteurs eux-mêmes chercheront peut-être à se prémunir contre l'épuisement en trichant avec le système (Hooper, 1993). Le système d'assurance qualité pourrait devoir faire face au problème classique du ritualisme qui guette tous les systèmes reposant trop lourdement sur des normes formelles que les usagers finissent par respecter mécaniquement en se désintéressant de plus en plus de l'atteinte des buts poursuivis (Merton, 1957). Le risque social du système est aussi celui de causer l'anémie des qualités favorisant l'esprit d'initiative au fur et à mesure que les acteurs apprennent à lui confier la protection contre leurs risques personnels (Bucio, 1993).

On retrouve, finalement, un risque politique associé au fait que les conséquences d'une défaillance du système seront normalement plus graves et subies par une collectivité plus grande que ce n'est le cas dans le régime traditionnel de la sous-traitance. L'échec de la coordination interfirmes sera durement ressenti non seulement par les partenaires immédiats, mais aussi par tous les acteurs du marché qui leur sont liés et par la collectivité environnante elle-même, s'il s'avère que l'organisation industrielle locale a été significativement influencée par la stratégie d'assurance qualité du donneur d'ordre. La conscience de ce risque amènera vraisemblablement les uns et les autres à réclamer qu'on leur concède un droit de participation à la régulation du système. Les acteurs locaux constateront alors que les systèmes-experts, du 
fait de leur complexité technique et de leurs liens avec l'environnement global d'où ils proviennent, échappent aux possibilités de contrôle direct des usagers (Giddens, 1990). Paradoxalement, la régulation des systèmes-experts fait appel, jusqu'à nouvel ordre, à des institutions de contrôle social qui sont, ellesmêmes, des systèmes-experts (Shapiro, 1987).

\section{Bibliographie}

AоKI, M. (1991), Économie japonaise. Information, motivations et marchandage, Paris, Economica, traduit par H.P. Bernard, p. 223-243.

ARChIER, G. et H. SÉRIEYX (1984), L'entreprise du $3^{e}$ type, Paris, Seuil.

ASANUMA, B. (1989), «Manufacturer-supplier relationships in Japan and the concept of relation-specific skill», Journal of the Japanese and International Economies, vol. 3, $\mathrm{n}^{\circ} 1$, p. 1-30.

BELLEY, J.G. (1991), «L'entreprise, l'approvisionnement et le droit. Vers une théorie pluraliste du contrat », Les cahiers de droit, vol. 32, nº 2, p. 253-299.

BELLEY, J.G. (1992), «Les transformations d'un ordre juridique privé. Les contrats d'approvisionnement à l'ère de la cybernétique et de la gestion stratégique», Les cahiers de droit, vol. 33, $\mathrm{n}^{\circ} 1$, p. 21-70.

BELLEY, J.G. (1993), «Contrat et citoyenneté. La politique d'achat régional d'une entreprise multinationale», Les cahiers de droit, vol. 34, no 3, p. 1063-1124.

Belley, J.G., A. Billette, L. Rasmussen et R.M. Thompstone (1995), «Moderniser la confiance. $L$ 'assurance qualité comme décontextualisation de la sous-traitance industrielle», La revue canadienne de sociologie et d'anthropologie, vol. 32, $\mathrm{n}^{\circ}$ 1, p. 1-23.

Billette, A., M. CARRIER et J. SAglio (1991), Structuration sociale d'un système industriel de PME: le cas de la région de St-Georges-de-Beauce, Sainte-Foy, Université Laval, $369 \mathrm{p}$.

BilletTe, A. et M. CARRIER (1993), "Régulation socio-identitaire des activités économiques beauceronnes », Recherches sociographiques, vol. XXXIV, $\mathrm{n}^{\circ} 2$, p. 261-277.

Bucio, F. (1993), «La sécurité phagocytaire», Dires, vol. 11, n 2, p. 133-154.

BURT, D.N. (1989), « Managing product quality through strategic purchasing», Sloan Management Review, printemps, p. 39-48.

ClouËT, P. (1989), Les achats, un outil de management, Paris, Les Éditions d'Organisation, p. 179-196.

DUBET, F. (1994), «Vraisemblance: entre les sociologues et les acteurs», L'année sociologique, $\mathrm{n}^{\circ} 44$, p. 83-107. 
Esposito, E. et M. RAFFA (1992), «Qualité et sous-traitance dans l'industrie italienne : quelques résultats d'une étude empirique», Revue internationale $P M E$, vol. 5, no 2, p. 57-82.

EYMARD-DUVERnAY, F. (1989), "Conventions de qualité et formes de coordination », Revue économique, vol. $40, \mathrm{n}^{\circ} 2$, p. 329-359.

FAVEREAU, O. (1989), « Marchés internes, marchés externes », Revue économique, vol. $40, \mathrm{n}^{\circ} 2$, p. $273-328$.

GidDEnS, A. (1990), The Consequences of Modernity, Stanford, Californie, ÉtatsUnis, Les Presses de l'Université Stanford, p. 36 ss, 92-111, 124 ss, 152.

HoOPER, C.H. (1993), «Bell-Québec: des employés autonomes », Mémo, octobrenovembre, cahier spécial, p. 18-19.

HoussiauX, J. (1957), «Le concept de quasi-intégration et le rôle des sous-traitants dans l'industrie », Revue économique, $\mathrm{n}^{\circ}$ 2, p. 221-247.

ITAMI, H. et S. SENBONGI (1992), « Competition by visible hand and subcontracting efficiency », Hitotsubashi University, ronéo, 40 p.

Julien, P.A. et M. MARChesnay (1988), La petite entreprise. Principes d'économie et de gestion, Ottawa/Paris, Éditions G. Vermette, Librairie Vuibert, p. 256.

Klein, B., R.G. Crawford et A.A. Alchian (1978), «Vertical integration, appropriable rents and the competitive contracting process», The Journal of Law and Economics, vol. XXI, n 2, p. 297-326.

LAmming, R. (1993), Beyond Partnership. Strategies for Innovation and Lean Supply, New York, Prentice-Hall, p. 140-211.

LEAVY, B. (1994), «Two strategic perspectives on the buyer-supplier relationship », Production and Inventory Management Journal, $2^{\mathrm{e}}$ trimestre, p. 47-51.

LEBORGNE, D. et A. LIPIETZ (1988), «L'après-fordisme et son espace », Les Temps Modernes, avril, $\mathrm{n}^{\circ}$ 501, p. 75-114.

LEENDERs, M.R. et D.L. BLENKHORN (1988), Reverse Marketing. The New BuyerSupplier Relationship, New York, The Free Press.

LEENDERS, M.R., H.E. FeARON et J. Nollet (1993), La gestion des approvisionnements et des matières, Boucherville, Gaëtan Morin Éditeur, p. 99-133, 171-221.

LORENZ, E.H. (1988), «Neither friends nor strangers: informal network of subcontracting in french industry », dans D. Gambetta (éd.), Trust. Making and Breaking Cooperative Relations, Oxford, Basil Blackwell, p. 194-210.

Luhmann, N. (1979), Trust and Power, Chichester, John Wiley and Sons, p. 26.

MAGNET, M. (1994), «The new golden rule of business », Fortune, 21 février, p. 60-64. 
MARTIN, Y. (1992), La sous-traitance au Québec, Montréal, Centre d'études en administration internationale (CETAI), Montréal, HEC, p. 195-198.

MAtThewS, P. (1993), "Getting the most out of value managed relationship », Modern Purchasing, octobre, p. 34-37.

Merton, R.K. (1957), Social Theory and Social Structure, 2e éd., Glencoe (Ill.), The Free Press, p. 184-187.

NolLET, J. et M.R. LeENDERS (1991), «Gestion des approvisionnements : horizon à court ou à long terme?», Gestion, novembre, p. 27-35.

Podolny, J.M. (1993), «A status-based model of market competition », American Journal of Sociology, vol. 98, $\mathrm{n}^{\circ} 4$, p. 829-872.

PRUS, R.C. (1989), Pursuing Customers. An Ethnography of Marketing Activities, Newbury Park, Sage Publications, p. 43-49.

RAMSAY, J. (1990), "The myth of the cooperative single source», Journal of Purchasing and Materials Management, hiver, p. 2-5.

RASMUSSEN, L. (1992), «Modernisation et formation de la main-d'œuvre : nouveaux rapports entre l'entreprise et le milieu régional. Le cas de la Stone-Consolidated de Ville de La Baie », Mémoire de maîtrise en études régionales, Université du Québec à Chicoutimi.

RAVIX, J.L. (1990), «L'émergence de la firme et des coopérations interfirmes dans la théorie de l'organisation industrielle: Coase et Richardson», Revue d'économie industrielle, vol. $51,1^{\mathrm{er}}$ trimestre, p. 27-51.

SAKo, M. (1992), Prices, Quality and Trust. Interfirm Relations in Britain and Japan, Cambridge, Cambridge University Press.

SChOnberger, R.J. (1982), Japanese Manufacturing Techniques. Nine Hidden Lessons in Simplicity, New York, The Free Press, p. 177.

SÉRIEYX, H. (1989), Le zéro mépris, Paris, Interéditions.

SHAPIRO, S.P. (1987), «The social control of impersonal trust », American Journal of Sociology, vol. 93, $\mathrm{n}^{0} 3$, p. 623-658.

SoUKUP, W.R. (1987), «Supplier selection strategies », Journal of Purchasing and Materials Management, été, p. 7-12.

ThOMPSTONE, R.M. (1991), «Gestion de la qualité des approvisionnements à l'Alcan », Université du Québec à Chicoutimi, Communication dans le cadre de la Semaine scientifique du Département des sciences économiques et administratives, 20 mars.

WiLliamson, O.E. (1979), «Transaction cost economics: the governance of contractual relations », The Journal of Law and Economics, vol. 22, p. 233-261. 
Williamson, O.E. (1985), «Assessing contract», Journal of Law, Economics and Organization, vol. 1, $\mathrm{n}^{\circ} 1$, p. 177-208.

XEROX (1993), «Dans la course à la qualité, il n'y a pas de ligne d'arrivée », Mémo, octobre-novembre, cahier spécial, p. 27. 